\title{
Desynapsis and Intragenomic Differentiation in Cultivated Species of Oryza
}

\author{
R. N. Misra and S. V. S. Shastry \\ Division of Genetics, Indian Agricultural Research Institute \\ New Delhi-12, India
}

Received April \&, 1967

Cultivated species of the genus Oryza, O. sativa and $O$. glaberrima were long recognised to belong to the same genome group AA (Morinaga 1959) based upon regular bivalent formation in interspecific hybrids involving these species. Exceptions to these generalizations have been pointed out by Yeh and Henderson (1962) who reported irregular pairing in one of the 10 crosses studied by them. Although the above workers proposed a distinct genome symbol EE for $O$. glaberrima, several workers disagreed with this symbolization not only for the reasons that chromosome pairing is influenced by several environmental factors but also that several of interspecific hybrids between the above species exhibit normal chromosome pairing. Hakim (1962) and Sampath (unpublished) reported that in a hybrid derived from one stock of $O$. sativa (Ac 2663) with O. glaberrima pairing was consistently low. These observations lead one to suspect the role of genic mechanisms suppression of chromosome pairing. Present study is, therefore, undertaken to investigate the stages of meiosis when failure of pairing occurs.

Fixed materials of two interspecific $F_{1}$ hybrids, O. glaberrima $\times 0$. sativa (Ac 806 ) and O. glaberrima $\times 0$. sativa (Ac 2663) were made available for cytological study through the kind courtesy of Sri. S. Sampath, Cytogeneticist, Central Rice Research Institute, Cuttack. The materials were fixed in 1:3 acetic alcohol with traces of ferric chloride and were squashed following aceto-carmine staining schedule. Meiotic data were secured from well spread preparations with clear differentiation.

\section{Results}

1) O. glaberrima $\times$ O. sativa (Ac 806) $-\mathrm{F}_{1}$

Chromosome pairing in this hybrid was normal with the following exceptions. It was difficult to analyse the chromosome pairing at pachytene due to stickiness of bivalents. While regular bivalent formation occurred in 30 PMCs at diplotene, in 2 PMCs 1 to 2 trivalents were recorded (Fig. 2). Seventeen PMCs were studied at metaphase I, of which 2 PMCs exhibited precocious separation leading to the formation of 2-4 univalents. Aberrant chiasma formation at metaphase I was 2.3 /bivalent. In majority of the PMCs at anaphase I sticky bridges and laggards were recorded (Fig. 1).

2) O. glaberrima $\times O$. sativa (Ac 2662) $-\mathrm{F}_{1}$

Pairing of chromosomes was found to be normal at pachytene in the 18 PMCs studied. Pairing at diplotene was exceedingly abnormal with almost complete univalents formation. In 8 out of 9 PMCs studied complete failure of pairing with 24 univalents was observed (Fig. 5). In one PMC 3 bivalents 


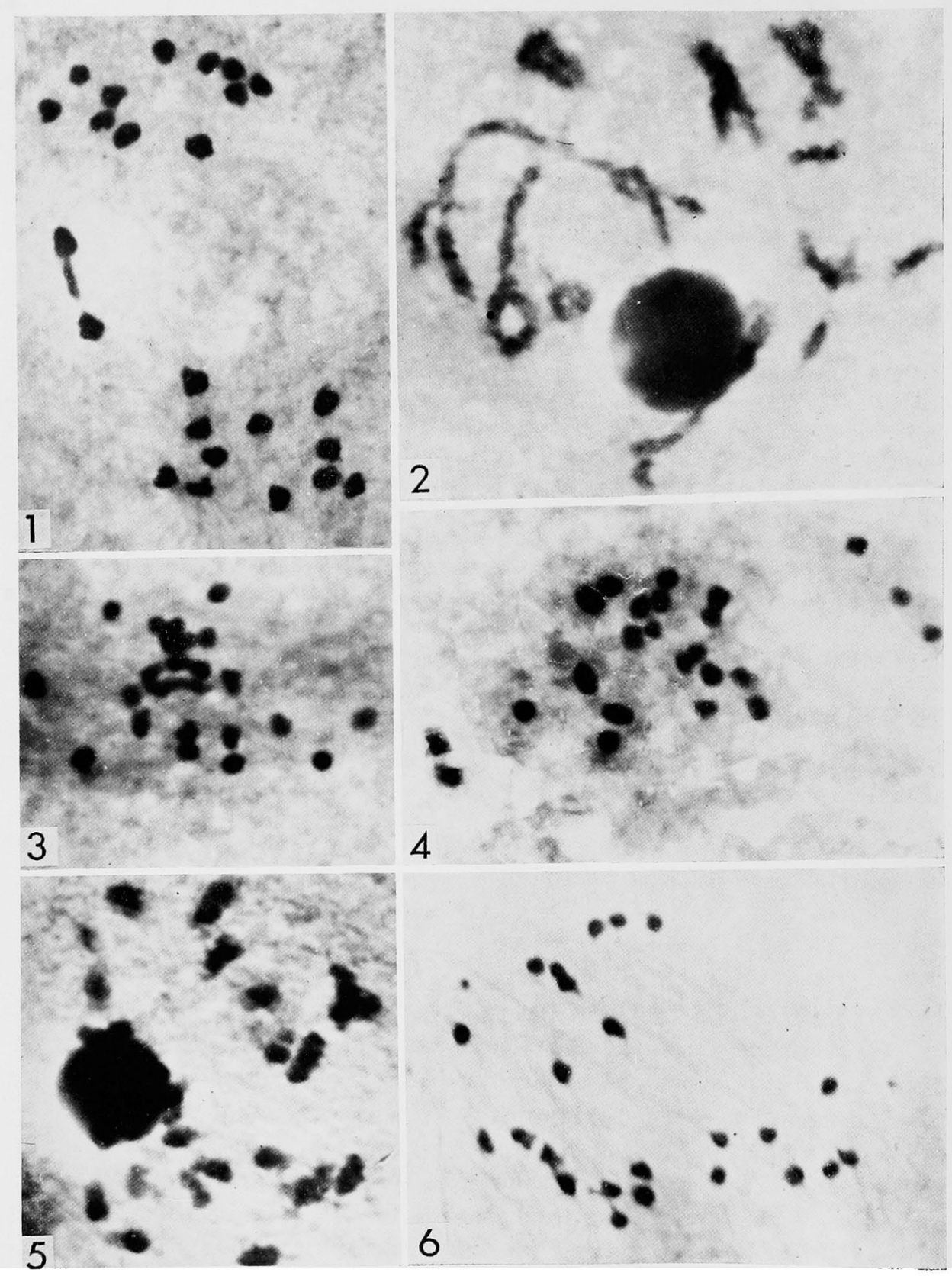

Figs. 1-6. 1-2, meiosis in 0. glaberrima $\times 0$. sativa $\left(A C\right.$ 806) $F_{1} .1$, anaphase I, showing a sticky bridge. 2 , diplotene, 2 trivalents ( $Y$-shaped and frying pan) +7 IIs +4 Is. 3-6, meiosis in 0 . glaberrima $\times 0$. sativa (AC 2663) $\mathrm{F}_{1} .3$, metaphase I, 2 II +20 Is. 4, metaphase I, one II +22 univalents (univalents associate side by side), 5 , diplotene, 3 IIs +18 Is. 6 , metaphase I, 24 Is. 
and 18 univalents were observed. Out of 26 PMCs studied at metaphase, 22 exhibited 24 univalents (Fig. 6). In the remaining PMCs, 1-2 bivalents were recorded (Figs. 3, 4). Most of the univalents at this stage remain associated side by side.

\section{Discussion}

Although chromosome pairing is due to homology, failure of bivalent formation in hybrids cannot always indicate the lack of homology between the parental genomes since failure of synapsis can possibly be brought about by many internal and external factors. Darlington (1937) reported a number of meiotic abnormalities which are controlled genetically. One of these, widely designated as desynapsis, is recognized by regular pairing at pachytene followed by the formation of univalents at later stage. Desynapsis is known to be controlled by one or a few pairs of genes (Beadle 1930, Richardson 1935, Prakken 1943, Li, Poa and Li 1945, Celarier 1955, Chheda and DeWet 1961), The significance of such a gene-controlled mechanisms for the synapsis of chromosomes in the diploidization of polyploids became evident following the classical work of Riley (1960).

Examples of such desynaptic mechanism are known in the genus Oryza which is supposed to have originated by polyploidy. Shastry, Sharma and Ranga Rao (1961) demonstrated in an interspecific hybrid between $O$. sativa and $O$. officinalis that pachytene pairing was normal followed by variable pairing at diplotene, diakinesis and complete univalent formation at metaphase $I$. This was interpreted by them as due to desynapsis rather than due to lack of true homology between the two constituent genomes of this hybrid.

Cytological analyses of the two interspecific hybrids between $O$. sativa and $O$. glaberrima reported here, showed variable types of chromosome pairing. In the hybrid, $O$. glaberrima $\times O$. sativa (Ac 806), pachytene pairing was normal while univalents and trivalents were observed in low frequency at diplotene and diakinesis. Metaphase I was normal with occasional precocious separation of bivalents. These observations indicate that the genomes of both the species are identical but differentiated by few chromosome structural changes. The meiosis in the second hybrid, O. glaberrima $\times O$. sativa (Ac 2663), is extremely interesting. Normal chromosome pairing is limited to pachytene stage followed by almost complete univalent formation at diplotene and metaphase $\mathrm{I}$. While in the former hybrid, the observation of higher chromosome association is indicative of chromosome structural changes, complete failure of pairing after pachytene in the latter one indicates the operation of genic mechanisms causing desynapsis.

Interspecific hybrids between $O$. sativa and $O$. glaberrima have been studied by Morinaga and Kuriyama (1957) who observed regular bivalent formation and designated the genomes of these two species as AA. While Richharia (1960), Nezu, Katayama and Kihara (1960), Morinaga (1963), Gopalakrishnan (1959) and Sharma (1964) unanimously recognized the same genome AA for all the 
species of $O$. sativa complex (including $O$. sativa and $O$. glaberrima), Yeh and Henderson (1962) proposed a separate genome for O. glaberrima and related African species since they observed complete univalent formation in one out of 10 sativa $\times$ glaberrima hybrids studied by them.

The observation of complete pairing failure in one out of the two hybrids reported here and the previously reported examples of one such case out of four hybrids studied by Hakim (1962) and one out of 10 cross-combinations studied by Yeh and Henderson (1962), may not be conclusive enough to recognize the two species under two district genome groups. Although $O$. sativa and $O$. glaberrima are closely related morphologically (Sharma 1964), karyomorphological data (Misra 1965) in diploids as well as meiosis in amphidiploids (Gopalakrishnan, Nayar and Sampath 1964) and genetic analysis of hybrid progenies (Richharia and Seetharaman 1962) indicate the role of chromosome structural changes differentiating these two species. Recovery of multivalents in low frequencies in the $F_{1}$ hybrids of $O$. glaberrima $\times O$. sativa (Ac 806) in the present study as well as those reported by Hakim (1962) further substantiate this view. In closely related species where gene exchange is restricted due to the erection of weak isolating barriers such as sterility factors and chromosome structural changes, sporadic gene mutations suppressing the pairing between these two sub-genomes may arise during phylogenetic differentiation. Distinct varietal difference in chromosome pairing of this interspecific hybrid indicates that desynapsis is gene-controlled. Therefore, in the light of the present observation, the genomes of $O$. sativa and $O$. glaberrima cannot be regarded as different.

\section{Summary}

Two hybrids between Oryza sativa and O. glaberrima were studied for chromosome pairing at meiosis. In both the hybrids pairing was normal at pachytene. In one of them, desynapsis results in low bivalent formation in diplotene and metaphase stages: The significance of these observations is discussed in relation to the nomenclature of genomes and phylesis.

Acknowledgement: We are grateful to Dr. M. S. Swaminathan for providing facilities. and kindly going through the manuscript.

\section{References}

Beadle, G. W. 1930. Genetical and cytological studies of Mendelian asynapsis in Zea mays. Cornell Agr. Expt. Sta. Mem. 129: 1-23.

Celarier, R. P. 1955. Desynapsis in Tradescantia. Cytologia 20: 69-83.

Chheda, H. R. and DeWet, J. M. J. 1961. Desynapsis in Bothriochloa hybrids. Proc. Okla. Acad. Sci. 41: 14-19.

Darlington, C. D. 1937. Recent Advances in Cytology. 2nd ed. J. and A. Churchill Ltd., London.

Gopalakrishnan, R. 1959. Cytogenetical studies on inter-specific hybrids in the genus Oryza. Associateship thesis, I. A. R. J. (Unpublished).

-, Nayar, M. N. and Sampath, S. 1964. Cytogenetics of two amphidiploids in genus Oryza. 
Euphytica 13: 57-64.

Jakim, K. L. 1962. Studies on crosses between cultivated and wild rices. M. Sc. Ag. Thesis (Unpubl.) of Division of Botany, Utkal Krushi-Mahabidalaya Bhubaneswar. i. H. W, Poa, W. K. and Li, C. L. 1945. Desynapsis in common wheat. Amer. J. Bot. 32: $92-101$.

Misra, R. N. 1965. Pachytene analysis in the genus Oryza. Ph. D. Thesis, I. A. R. I. New Delhi-12.

Morinaga, T. 1959. Note on genom analysis in Oryza species. Intern. Rice Comm. News, Letter 8(3): 10-11.

- 1963. Cytological investigation on Oryza species. Symp. Rice Genet. and Cytologenet. Los Banos, Laguna, Philippines.

- and Kuriyama, H. 1957. Cytogenetical studies on Oryza sativa L. IX. The $F_{1}$ hybrid of $O$. sativa L. and $O$. glaberrima Steud. Jap. J. Breed. 7: 57-65.

Nezu, M., Katayama, T. C. and Kihara, H. 1961. Genetic study of the genus Oryza. 1. Crossibility and chromosomal affinity among 17 species. Seiken Ziho 11: 1-11.

Prakken, R. 1943. Studies of a synapsis in rye. Hereditas 29: 475-495.

Richardson, M. M. 1935. Meiosis in Crepis II. Failure of pairing in Crepis capillaris. J. Genet. 31: 119-143.

Richharia, R, H. 1960. Origins of cultivated rices. Indian J. Genet. 20: 1-14.

- and Seetharaman, R. 1962. Studies in Oryza glaberrima Steud. -J. Inheritance of colour in apiculus and stigma, inter-relationship of genes and their significance. The Nucleus 5: 87-94.

Riley, R. 1960. The diploidization of polyploid wheat. Heredity 15: 407-430.

Sharma, S. D. 1964. Interspecific relationship in the genus Oryza L. Unpublished Ph. D. Thesis, Indian Agricultural Research Institute, New Delhi.

Shastry, S. V. S., Sharma, S. D. and Ranga Rao, D. R. 1961. Pachytene analysis in Oryza. III. Meiosis in an inter-sectional hybrid. O. sativa $\times O$. officinalis. The Nucleus 4: $67-80$.

Yeh, B. and Henderson, M. T. 1962. Cytogenetic relationships between African annual diploid species of Oryza and cultivated rice, O. sativa L. Crop Sci. 2: 463-467. 